\title{
TIME SCALE OF RADIO SOURCES ACTIVITY FROM THE STATISTICAL MODEL OF VARIABILITY
}

\author{
P. MAGDZIARZ and J. MACHALSKI \\ Astronomical Observatory, Jagiellonian University \\ ul. Orla 171, \\ PL-30244 Cracow, Poland
}

The lack of a plateau in the average structure function (hereafter SF) for the complete flux-limited sample of Seielstad et al. (1983) [Fig.1] suggests that the mean time scale of radio sources variability at $10.8 \mathrm{GHz}$ should be longer than the 3.8-yr time base of those observations. A comparison of the variability time scales derived from the SF analysis with those predicted by the statistical model of Magdziarz \& Machalski (1993, AA, in print) [Fig.2] confirms consistency of that model. The mean time scale of variability of $\langle\tau\rangle=6 \pm 2$ yr was found in both cases for $\nu=10.8 \mathrm{GHz}$.

The mean variability time scale characterizing the total population of radio sources seems to be longer than that (about $2 \mathrm{yr}$ ) commonly accepted from observations of selected variables (e.g. Altschuler 1989, Fund.Cosm.Phys. 14,37; Hughes et al. 1992, ApJ 396,469). This is consistent with the selection effects if the shot noise character of variability (Cruise \& Dodds 1985, MNRAS 215,417; Hughes et al. 1992, ApJ 396,469), giving a wide range of time scales, is taken into account, and means that many sources can likely vary at time scales exceeding 40-50 yr. The linear character of intermediate time-lags SF found by Hughes et al. for UMRAO sources agrees with predictions of our statistical model, and confirms the universal physical nature of the variability. This is also consistent with predictions of the standard shock model of Marsher \& Gear 1985, ApJ 298,114 (cf. also Valtaoja et al. 1992, AA 254,71).
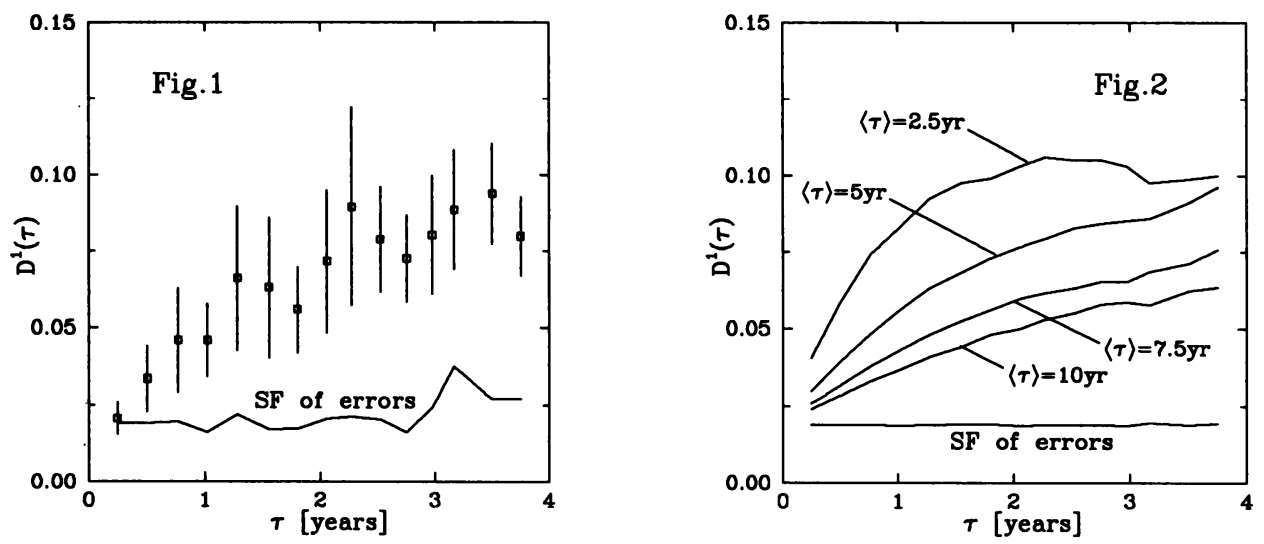

398

T. J.-L. Courvoisier and A. Blecha: Multi-Wavelength Continuum Emission of AGN, 398.

(C) 1994 IAU. Printed in the Netherlands. 